\title{
P-mode oscillation on slim discs
}

\author{
Li Xue and Ju-Fu Lu \\ Department of Astronomy and Institute of Theoretical Physics and Astrophysics, \\ Xiamen University, Xiamen, Fujian 361005, China \\ email: lixue@xmu.edu.cn
}

\begin{abstract}
We numerically investigate the thermally unstable accretion discs around spinning black holes with different spins. We adopted an additional evolutionary viscosity equation to replace the standard alpha-prescription based on the results of two MHD simulations. We find an interesting oscillation when accretion switches to slim disc mode. The oscillation arises from the sonic point of accretion flow and propagates outwards. We mimic the bolometric light-curve and find a series of harmonics on its power spectrum. The frequency ratio of those harmonics is a regular integer series. The lowest frequency of the harmonics is identical to the prediction of trapped p-mode in QPO theory.
\end{abstract}

Keywords. accretion, accretion disks, gravitation, relativistic processes, stars: black holes, Xrays: bursts

\section{Introduction}

High-frequency quasi-periodic oscillations (HFQPOs) have been observed in some black hole (BH) X-ray binaries, which only appear in "steep power law" state in high luminosity $\left(L>0.1 L_{\mathrm{Edd}}\right)$ and are in range of 40 to $450 \mathrm{~Hz}$. These frequencies are comparable to the orbital frequency of innermost stable circular orbit (ISCO) of a stellar-mass BH. It is believed that the mechanism behind them are closely related to the dynamics of inner regions of $\mathrm{BH}$ accretion discs (see Remillard \& McClintock 2006, Kato et al. 2008, Belloni et al. 2012, for reviews).

In Xue et al. (2011), we have described a code and relevant equations for the axisymmetric relativistic accretion flows around spinning black holes. The viscosity in that code is described by the standard alpha-prescription (Shakura \& Sunyaev 1973). In this work, we update the code with adopting an additional evolutionary viscosity equation to replace the alpha-prescription based on two MHD simulations, Hirose et al. (2009) and Penna et al. (2013). We run the new version code for several thermally unstable accretion models and we find an interesting oscillation when the accretion switches to slim disc mode within the inner disc region. We argue that this oscillation is the trapped p-mode, which is excited by the sonic-point instability on a transonic accretion flow across ISCO (Kato 1978). Indeed, our new code represent a time-dependent accretion model that is able to explain the observational HFQPOs.

\section{Results}

In fact, the most essential feature in our models is the evolution of viscosity. It reasonably introduces a time-delay between the viscous stress and total pressure, but this delay vanishes in the alpha-prescription. Our colleagues Lin et al. (2011) and Ciesielski et al. (2012) have focused on the effects of this time-delay on thermal instability and they conclude that it can not remarkably diminish or remove the instability for typical 


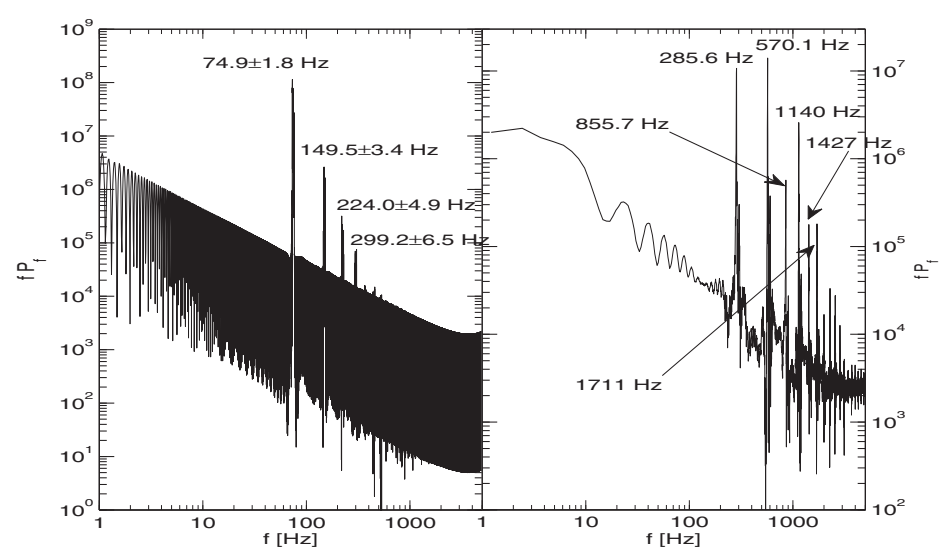

Figure 1. PSDs of light-curves. The left panel is for the disc around a non-spinning black hole $\left(a^{*}=0, M=10 M_{\odot}, n=1\right)$. The right panel is for the disc around a fast-spinning black hole $\left(a^{*}=0.947, M=7.02 M_{\odot}, n=4\right)$.

delay lengths. Indeed, in this work, we find and investigate the other effect of this delay on the oscillation of accretion discs (please concern our paper in ApJLett for the detail).

Here, we only show the power spectral densities (PSDs) of oscillating light-curves for two typical models (around non-spinning and fast-spinning black holes) in Figure 1. Their oscillations both arise in high luminosity state $\left(L \gtrsim 0.2 L_{\mathrm{Edd}}\right)$. The fundamental frequency (the lowest frequency of harmonics) is $\sim 74.9 \mathrm{~Hz}$ for non-spinning model and $\sim 285.6 \mathrm{~Hz}$ for fast-spinning model, which are both identical to the predicted $71.3 \mathrm{~Hz}$ and $300 \mathrm{~Hz}$ (see Kato et al. 2008). It is a remarkable feature, in Figure 1, that there are many harmonics on PSDs and the frequency ratio is a regular integer series $1: 2: 3 \ldots$ This interesting feature is potentially useful for explain the observational QPO pairs, but the analogue observation with our numerical results is not straight and convenient. It needs to carefully consider the gravitational red-shift and bending on the emitted photon. However, for convenience, we only construct the light-curves by directly integrating the radiation cooling flux at each radius in this work.

\section{Acknowledgements}

This work was supported by the National Natural Science Foundation of China under grants 11233006 and 11373002

\section{References}

Belloni, T. M., Sanna, A., \& Méndez, M. 2012, MNRAS, 426, 1701

Ciesielski, A., Wielgus, M., Kluźniak, W., Sądowski, A., Abramowicz, M., Lasota, J.-P., \& Rebusco, P. 2012, A\& A, 538, 148

Hirose, S., Krolik, J. H., \& Blaes, O. 2009, ApJ, 691, 16

Kato, S. 1978, MNRAS, 185, 629

Kato, S., Fukue, J., \& Mineshige, S. 2008, Black-Hole Accretion Disks: Towards a New Paradigm (Kyoto: Kyoto Univ. Press), p. 422

Lin, D.-B., Gu, W.-M., \& Lu, J.-F. 2011, MNRAS, 415, 2319

Penna, R. F., Sądowski, A., Kulkarni, A. K., \& Narayan, R. 2013, MNRAS, 428, 2255

Remillard, R. A. \& McClintock, J. E. 2006, ARAA, 44, 49

Shakura, N. I. \& Sunyaev, R. A. 1973, A\& A, 24, 337

Xue, L., Sądowski, A., Abramowicz, M. A., \& Lu, J.-F. 2011, ApJS, 195, 7 